Research Paper

\title{
Fuzi Enhances Anti-Tumor Efficacy of Radiotherapy on Lung Cancer
}

\author{
Qi Zhang1, Xue Chen ${ }^{1}$, Youjun Luo ${ }^{1}$, Huaping Ren², Tiankui Qiao ${ }^{1 凶}$ \\ 1. Department of Oncology, Jinshan Hospital, Fudan University, Shanghai 201508, China; \\ 2. Department of Chinese Medicine, Jinshan Hospital, Fudan University, Shanghai 201508, China. \\ $\triangle$ Corresponding author: Tiankui Qiao, Department of Oncology, Jinshan Hospital, Fudan University, Shanghai 201508, China. Tel +86-18930778786; E-mail: \\ qiaotk@163.com \\ (C) Ivyspring International Publisher. This is an open access article distributed under the terms of the Creative Commons Attribution (CC BY-NC) license \\ (https://creativecommons.org/licenses/by-nc/4.0/). See http://ivyspring.com/terms for full terms and conditions.
}

Received: 2017.07.30; Accepted: 2017.08.25; Published: 2017.10.23

\begin{abstract}
In traditional Chinese medicine, Fuzi is widely used as an antitumor agent or an adjuvant medication combined with radiotherapy and chemotherapy, but its mechanism remains unclear. In this study, we investigated anti-tumor and immunoregulation efficacy of Fuzi combined with radiotherapy in mice with Lewis lung cancer (LLC). We found that Fuzi combined with radiotherapy significantly inhibited the growth of LLC, promoted the apoptosis of cancer cells, and prolonged the survival of mice with LLC. Mechanistically, we found that Fuzi decreased the proportion of Treg cells, reduced serum levels of cytokines such as interleukin (IL)-10 and transforming growth factor (TGF)- $\beta$, and downregulated the expression of programmed death ligand-1 in mice with LLC subjected to radiotherapy. This study suggests that Fuzi has immunomodulation function to act as radiosensitizer and improve radiotherapy against lung cancer.
\end{abstract}

Key words: Radiotherapy, Fuzi, TGF- $\beta$, PD-L1, Treg cells, Lung cancer.

\section{Introduction}

Lung cancer is now the leading cause of cancer deaths in both men and women in the United States, accounting for $26 \%$ of cancer deaths in 2017 [1]. Lung cancer is also the leading cause of cancer death in men and the second leading cause of cancer death in women worldwide, with non-small cell lung cancer (NSCLC) accounting for approximately $80 \%$ of all cases [2,3]. Radiotherapy has become an important treatment modality for medically inoperable patients with NSCLC [4]. However, studies have shown that the proportion of regulatory $\mathrm{T}$ cells (Tregs) in the body and the expression of programmed death ligand-1 (PD-L1) in tumor microenviroment are increased after radiation, reducing the efficacy of radiotherapy $[5,6]$. Therefore, appropriate immunomodulators capable of reversing or preventing damage to the immune system would be important to improve the efficacy of tumor radiotherapy.

The lateral roots of Aconitum carmichaelii Debx. (Ranunculaceae), also named "Fuzi", are used in raw or prepared forms in Chinese medicine. Currently, 122 chemical constituents have been isolated and identified from Fuzi and most of them are alkaloids [7]. Fuzi has long been used to treat heart failure, shock, pulmonary hypertension, coronary heart disease, and rheumatic heart disease for its analgesic and anti-inflammatory effects [8, 9]. In addition, recent studies indicate that Fuzi inhibits tumor growth in vivo by improving host immune responses $[10,11]$. However, anti-tumor efficacy of Fuzi in combination with radiotherapy remains unclear. In this study, we aimed to evaluate anti-tumor and immunomodulatory efficacy of Fuzi in radiotherapy of lung cancer using mouse with Lewis lung cancer as the experimental model.

\section{Materials and Methods}

\section{Cell culture and reagents}

Lewis lung cancer (LLC) cell line was purchased from Cell Bank of Shanghai Institute of Cell Biology, 
Chinese Academy of Science, and cultured in Dulbecco's Modified Eagle's Medium (DMEM; Gibco, USA) supplemented with $10 \%$ fetal bovine serum. The cells were maintained at $37^{\circ} \mathrm{C}$ in a humidified atmosphere containing $5 \% \mathrm{CO}_{2}$.

Fuzi was provided by the Jinshan Hospital of Fudan University (Shanghai, China) and identified by associate chief physician, Huaping Ren, Department of Chinese medicine, Jinshan Hospital of Fudan University. Fuzi extract was prepared as follows: Fuzi was crushed into coarse powder, soaked in a 10-fold volume of water for $30 \mathrm{~min}$ and then boiled for $2 \mathrm{~h}$. The supernatant was concentrated after filtration using $0.22-\mu \mathrm{M}$ filters. Freeze-dried powder was then prepared and stored at $4^{\circ} \mathrm{C}$.

\section{Mice}

Female C57BL/6J mice were provided by the Shanghai Experimental Animal Center and maintained in a specific pathogen-free-grade animal room. The mice were inoculated with $2 \times 10^{6}$ fresh LLC cells in the right leg when they reached 6-8 weeks of age and weighed 18-22 g. Ten days after the inoculation of tumor cells, the mice were randomly divided into four groups $(\mathrm{n}=10)$. Group I received saline solution (N.S., $5 \mathrm{ml} / \mathrm{kg}$ ) as a control (C group). Group II received radiation (5 Gy) every day for 5 days from day 4 to day 8 (irradiation group; IR). Group III received Fuzi at a dose of $3.60 \mathrm{mg} / 10 \mathrm{~g}$ daily from day 1 to day 11 (Fuzi group). Group IV was pretreated with Fuzi and irradiated as described above (IR + Fuzi). All mice were fasted for $30 \mathrm{~min}$ after intragastric administration. Mice in IR group were tied to small boards, and their tumors were centered in a 3-cm2 field. Radiation was delivered five times to the tumor-bearing leg using a linear X-ray accelerator unit with a single dose of 5 Gy from day 4 to day 8, for a total dose of 15 Gy. Fuzi extracts or saline solution were administered to $\mathrm{C} 57$ mice by intraperitoneal injection. Ten mice in each group were observed for tumor growth and survival. Starting on the 16th day, tumor-bearing mice in each group were euthanized and sacrificed by intraperitoneal injection of a lethal dose of pentobarbital $(200 \mathrm{mg} / \mathrm{kg}$ body weight). The tumor volume was measured when tumors were visible, and survival curves for the ten mice from each group were surveyed for 80 days after inoculation. Tumor volume was determined by the following formula: tumor volume $\left(\mathrm{cm}^{3}\right)=1 / 2 \times$ length $(\mathrm{cm}) \times$ width $^{2}(\mathrm{~cm})$. Tumor inhibitory rate was calculated as follows: Tumor inhibitory rate $=1-$ (Mean tumor weight of treated group/mean tumor weight of control group) $\times 100 \%$. All protocols were carried out in accordance with the ethics committee of Jinshan Hospital (Shanghai, China).

\section{TUNEL}

Tumor samples were dissected from sacrificed mice and stained using TdT-mediated dUTP nick end labeling (TUNEL) kit (KeyGen, Jiangsu, China). The stained samples were observed under a microscope by two pathologists independently. Approximately 1,500 cells in total were counted in three high-power microscope fields. The apoptosis rate was calculated as follows: (number of apoptosis cells/total number of cells) $\times 100 \%$.

\section{ELISA}

On the $16^{\text {th }}$ day, serum was collected from the mice and serum IL-2, IL-6, IL-12 and IL-10 levels were analyzed using Mouse Th17 Milliplex Map kit, and TGF- $\beta$ level was analyzed using Mouse TGF- $\beta$ Milliplex Map kit (Millipore, Billerica, MA, USA). The results were measured by a Bio-Plex MAGPIX Multiplex Reader (Bio-Rad Laboratories, Hercules, CA, USA).

\section{Flow cytometry}

Spleens were ground in RPMI 1640 medium (HyClone, MA, USA) and then passed twice through 200 mesh nylon. Erythrocytes were removed using Red Blood Cell Lysis Buffer. The cell suspension was then filtered through mesh, and the number of lymphocytes was counted under a microscope. Tregs were phenotyped as $\mathrm{CD}^{+}{ }^{+} \mathrm{Foxp}^{+}$as follows: the cells were stained with CD4-percp and Foxp3-PE (eBioscience, San Diego, CA, USA) and subjected to cytometry on a FACSCalibur flow cytometer (Becton Dickinson). $\mathrm{CD}^{+}$cells and $\mathrm{CD} 4{ }^{+} \mathrm{Foxp}^{+}$cells were quantified.

\section{RT-PCR}

Total RNA was extracted using Trizol reagent (Invitrogen, USA). cDNA was synthesized from total RNA and amplified using SYBR Premix TaqTMII kit (Takara, Dalian, China). The sequences of the primers were as follows: $\beta$-actin $5^{\prime}$-CATCCGAAAGACCTCT ATGCCAAC-3', 5' -ATGGAGCCACCHATCCACA-3'; PD-L1: 5' -AGCGAATVACGCTGAAAGTCAA-3', 5'-GGATAACCCTCGGCCTGACA-3'. Gene amplifycation was performed as follows: $95^{\circ} \mathrm{C}$ for $30 \mathrm{~s}(1$ cycle); $95^{\circ} \mathrm{C}$ for $5 \mathrm{~s}, 60^{\circ} \mathrm{C}$ for $32 \mathrm{~s}\left(40\right.$ cycles); and $60^{\circ} \mathrm{C}$ for 1 min (1 cycle).

\section{Western blot analysis}

Tumor tissue was homogenized in cold lysis buffer containing $1 \mathrm{mM}$ phenylmethane sulfonyl fluoride and lysed for at least $30 \mathrm{~min}$ on ice. Protein concentrations were measured using the Blue Fast Staining Solution (Beyotime, Shanghai). Equal amounts $(40 \mu \mathrm{g})$ of protein were separated via $10 \%$ 
SDS-PAGE and transferred to PVDF membranes (Millipore). Membranes were blocked with TBS/T containing 5\% skim milk for $1 \mathrm{~h}$ and incubated overnight at $4^{\circ} \mathrm{C}$ with PD-L1 antibody and a-tubulin antibody (Abcam, UK). The membranes were then incubated with secondary antibodies (Beyotime Institute of Biotechnology, Jiangsu, China) at room temperature for $2 \mathrm{~h}$. After several washes with TBS/T, the blots were detected using Immobilon ${ }^{\mathrm{TM}}$ Western Chemiluminescent HRP Substrate (Millipore) and quantified using a Tanon-4500 Gel Imaging System (Tanon Science \& Technology Co., Ltd.).

\section{Statistical analysis}

Statistical analysis was performed using SPSS13.0 software. The data were expressed as mean \pm standard derivation (SD). The comparison among multiple groups was analyzed by analysis of variance and the comparison between two groups was analyzed by $\mathrm{t}$ test. $\mathrm{P}<0.05$ was considered statistically significant.

\section{Results}

\section{Fuzi enhanced anti-tumor efficacy of irradiation in LLC mouse model}

C57BL/6J mice were challenged with LLC cells and then treated with Fuzi and irradiated. We observed significantly slower tumor growth in IR + Fuzi group compared to other three groups on the $9^{\text {th }}$, $11^{\text {th }}$ and $13^{\text {th }}$ day $(p<0.05)$ (Fig. 1A). As shown in Fig. $1 \mathrm{~B}$, the median survival time of mice in the control, IR, Fuzi, and IR + Fuzi group was 30, 51.5, 49, and 63 days, respectively. The difference between IR + Fuzi and control groups was significant $(p=0.0001)$. The tumor volume was obviously the smallest in IR + Fuzi group among all four groups (Fig. 1C). The tumor weight was the lowest and tumor inhibition rate was the highest IR + Fuzi group among all four groups (Fig. 1D). Collectively, these results suggest that Fuzi enhances anti-tumor efficacy of irradiation in LLC mouse model.

A

C

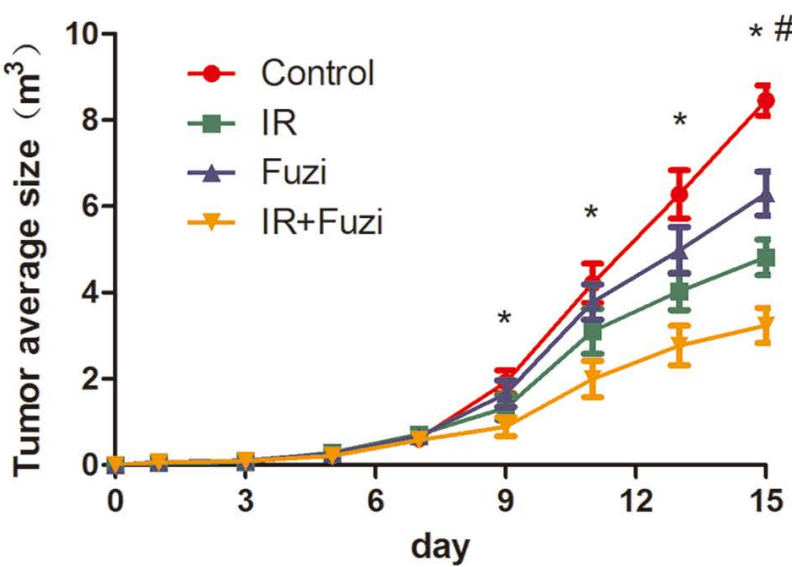

B
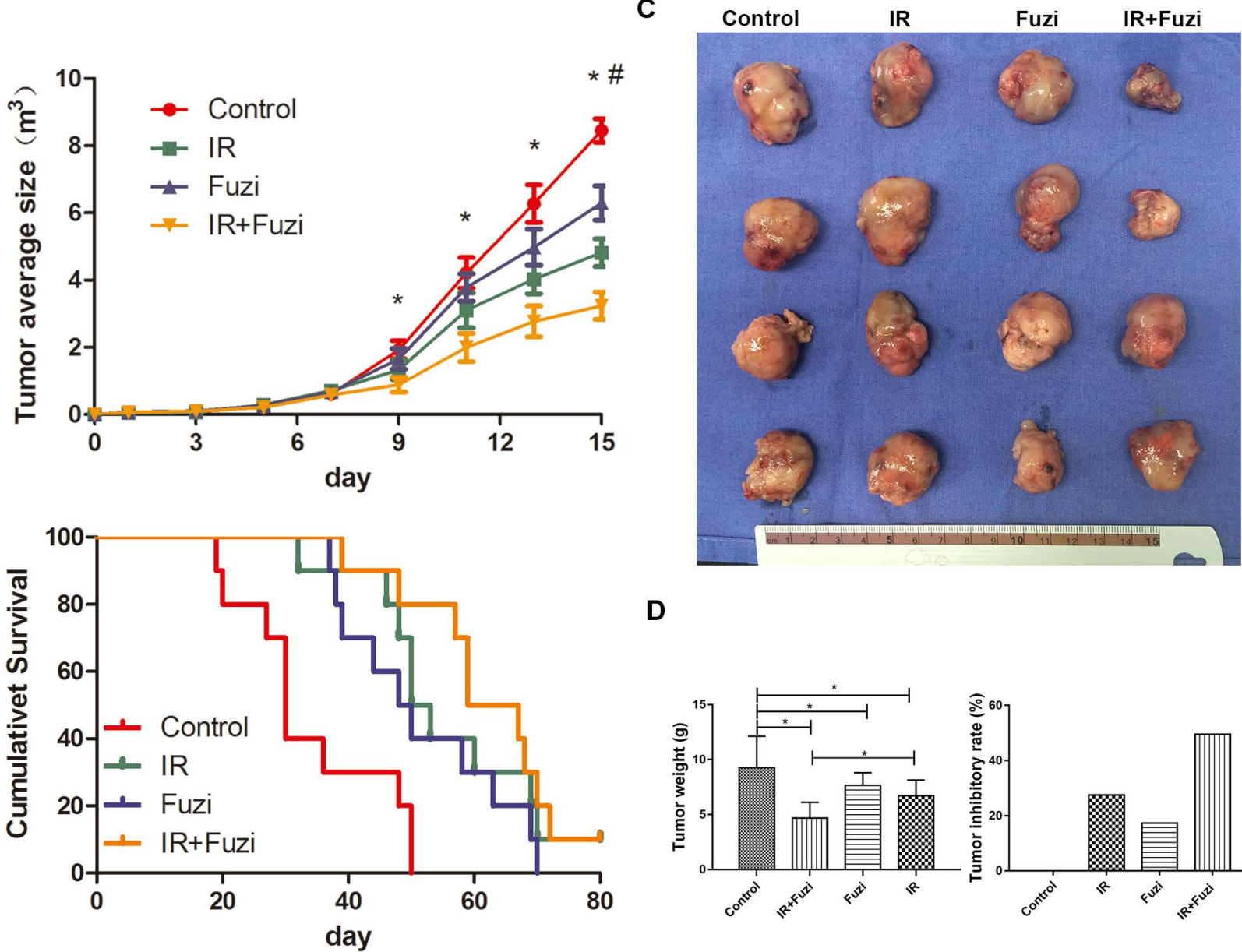

D

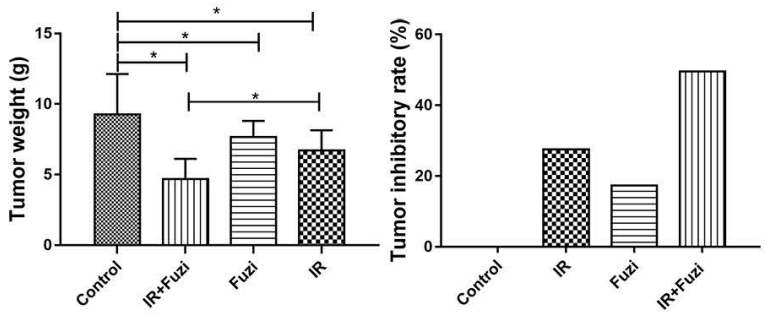

Figure 1. Enhanced anti-tumor effects of irradiation combined with Fuzi. A. Tumor growth curve. ${ }^{*} p<0.05$ vs. control group, \# $p<0.05$ vs. IR group. B. Kaplan-Meier plot of the estimated survival for the mice in four groups. Survival in IR + Fuzi group was significantly better than the other three groups $(p<0.01)$. $\mathbf{C}$. Size distribution of the tumors in four groups. D. Tumor weight and tumor inhibitory rate in four groups. Tumor weight was the lowest and tumor inhibitory rate was the highest in IR + Fuzi group. ${ }^{*} p<0.05$. Data represented mean $\pm S D(n=5)$. 


\section{Fuzi enhanced irradiation induced tumor cell apoptosis in LLC mouse model}

The apoptosis of xenograft cells was assessed by TUNEL staining. Representative TUNEL staining images in four groups were shown in Fig. 2A-D. Quantitative analysis showed that the apoptosis rate was the highest in IR + Fuzi group, significant higher than in other groups $(p<0.05$, Fig. 2E). These results suggest that Fuzi could enhance irradiation induced tumor cell apoptosis in LLC mouse model.

\section{Fuzi changed serum levels of cytokines in LLC mouse model}

The secretion of cytokines in LLC mouse model was assessed by ELISA. As shown in Figure 3, IL-2, IL-5, IL-6, IL-12 levels were significantly higher while IL-10 and TGF- $\beta$ levels were significantly lower in IR + Fuzi group compared to other three groups $(p<$
$0.05)$. These results indicate that Fuzi could increase IL- 6 and IL-12 secretion and reduce TGF- $\beta$ secretion in LLC mouse model.

\section{Fuzi reduced radiation induced increase in Treg cell fraction}

Radiation-induced changes in the number and ratio of $\mathrm{CD}^{+}{ }^{+} \mathrm{Foxp}^{+}$Treg cells were investigated in lymphocytes isolated from the spleens of irradiated mice. Representative flow cytometry images of Treg cells in four groups were shown in Fig. 4A. Quantitative analysis showed that $\mathrm{CD}^{+}{ }^{+}$Foxp $3^{+}$Treg cell ratio increased in IR group compared to control group, but Fuzi treatment could reduce $\mathrm{CD}^{+}{ }^{+}$Foxp3 ${ }^{+}$Treg cell ratio (Fig. 4B). These results indicate that Fuzi could antagonize radiation induced Treg accumulation in LLC mouse model.
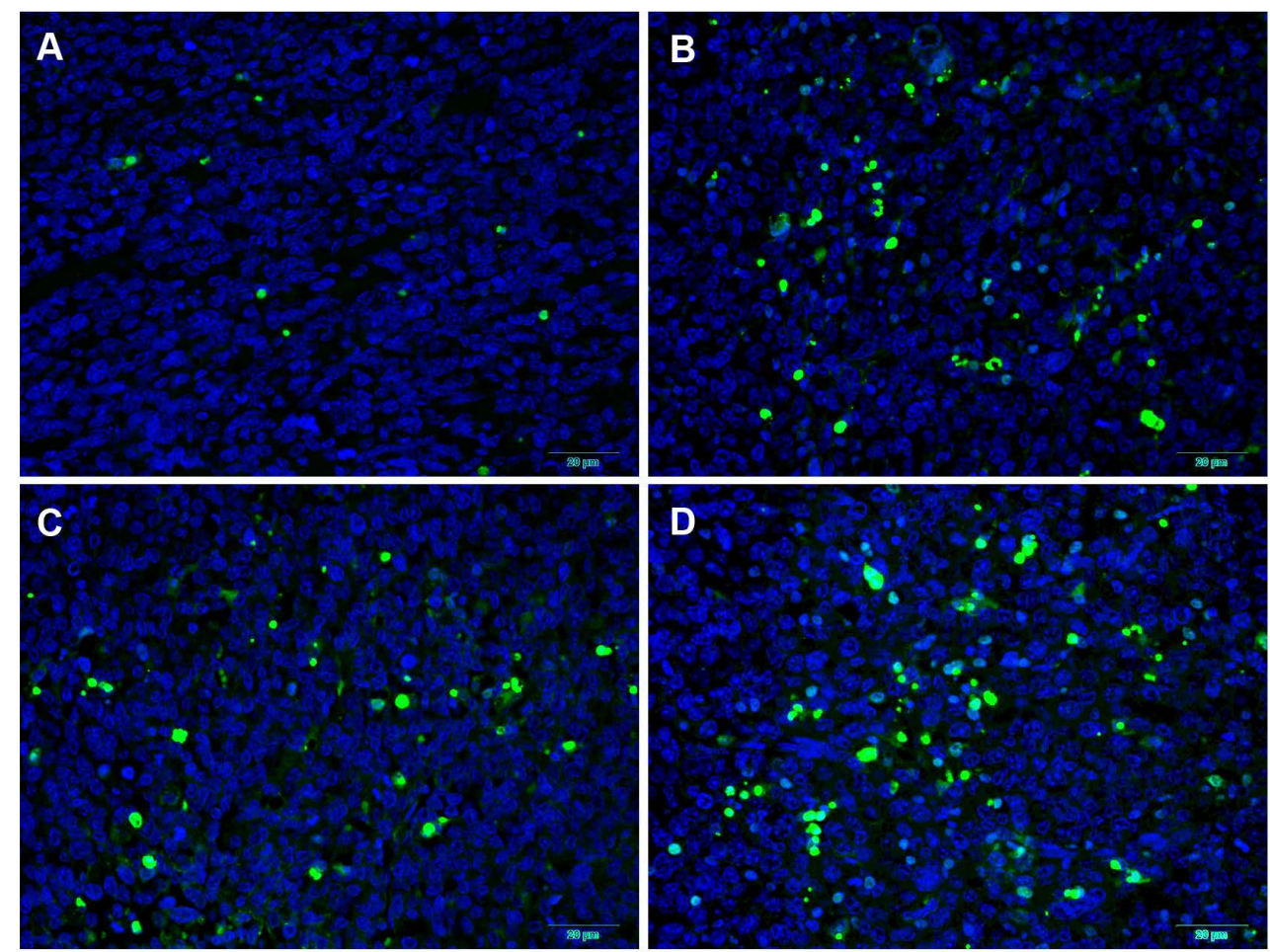

$\mathbf{E}$

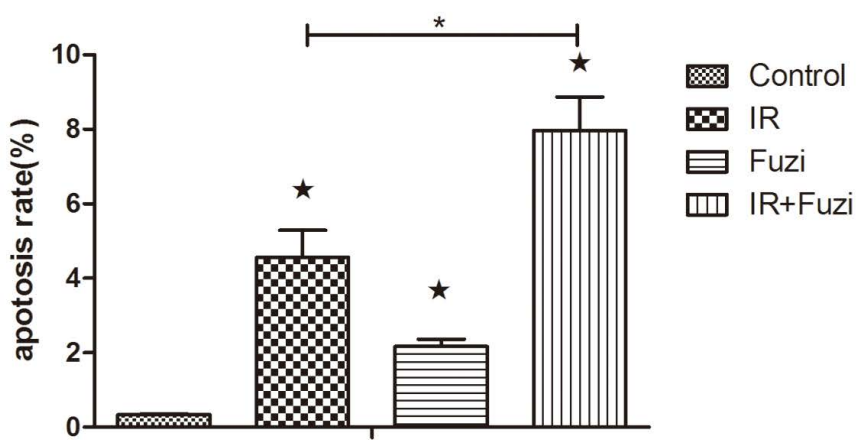

Figure 2. Fuzi promotes lung cancer cell apoptosis. A-D. Representative TUNEL staining of apoptotic cells in Control, IR, Fuzi, and IR+Fuzi group, respectively (Scale bar: $20 \mu \mathrm{m})$. E. The apoptosis rate in four groups. The apoptosis rate was the highest in IR+Fuzi group. $\star p<0.05$ vs. Control group. $* p<0.05$ for IR + Fuzi vs. IR group. Data represented mean \pm SD $(n=3)$. 

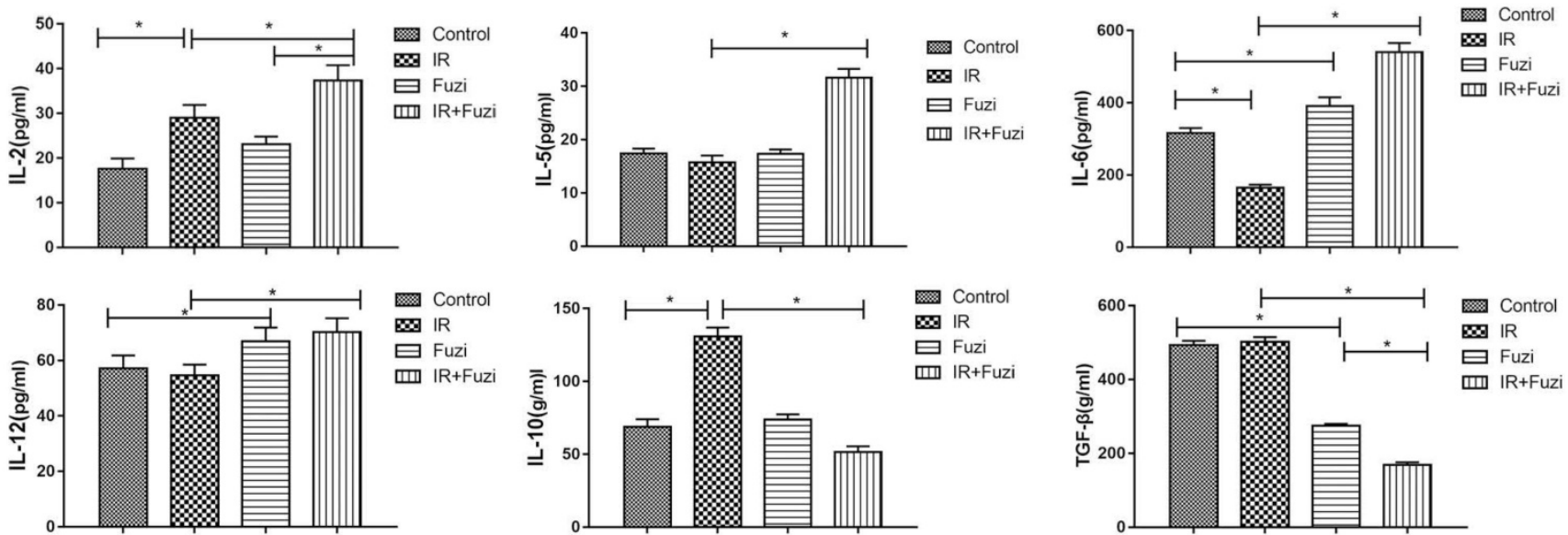

Figure 3. Serum levels of IL-2, IL-5, IL-6, IL-12, IL-10 and TGF- $\beta$ in four groups of mice. ${ }^{*} p<0.05$. Data represented mean \pm SD $(n=5)$

A
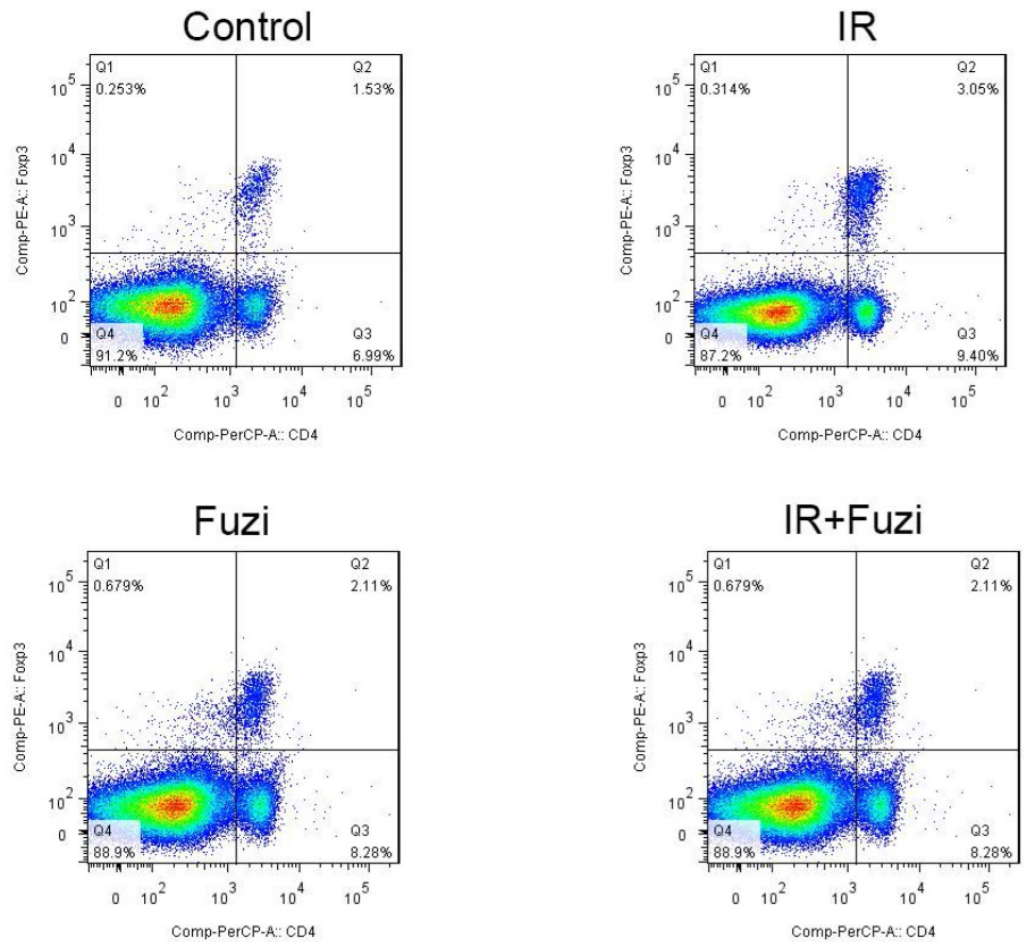

B

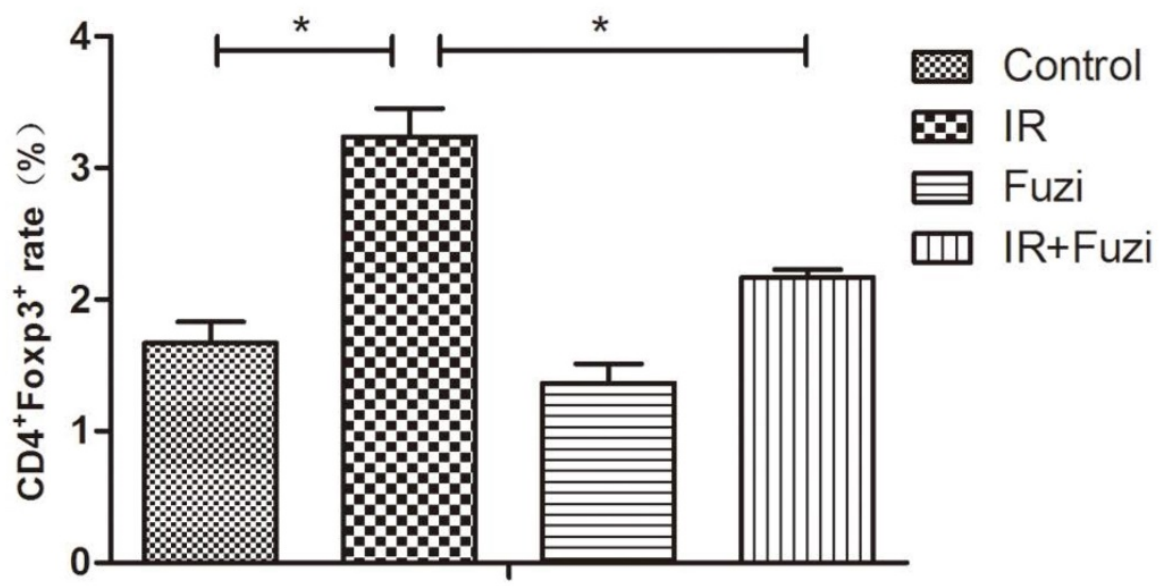

Figure 4. The proportion of $C D 4^{+}$Foxp $3^{+}$Treg cells in the spleens of four groups. A. Representative flow cytometry images of CD4+Foxp $3^{+}$Treg cells in each group. B. The $C D 4^{+}$Foxp $3^{+}$Treg rate in four groups. ${ }^{*} p<0.05$. Data represented mean $\pm \operatorname{SD}(n=3)$. 


\section{Fuzi reduced radiation induced upregulation of PD-LI in LLC mouse model}

The expression of PD-L1 mRNA in xenograft tissues were detected by RT-PCR. As shown in Fig. 5A, while PD-L1 mRNA expression increased after IR, it decreased significantly after additional Fuzi treatment $(p<0.05)$. Consistently, Western blot analysis showed that PD-L1 protein level increased after IR, but decreased significantly after additional Fuzi treatment $(p<0.05$, Fig. 5B, C). These results indicate that Fuzi could inhibit radiation induced upregulation of PD-L1 in LLC mouse model.
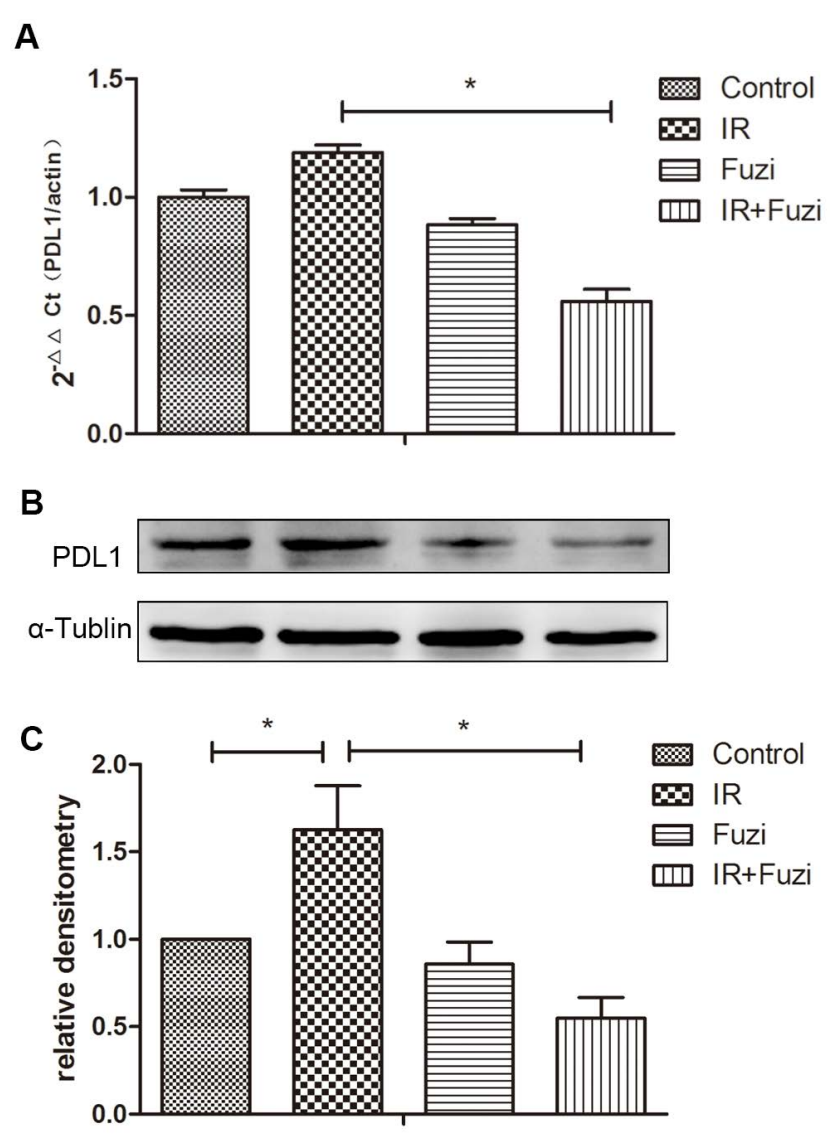

Figure 5. PD-L1 expression in tumor tissues of four groups. A. RT-PCR analysis of PD-LI mRNA levels in the transplanted tumor cells. Data represented mean $\pm \operatorname{SEM}(n=3)$. ${ }^{*} p<0.05$. B. Western blot analysis of PD-LI protein levels in the transplanted tumor cells. Shown were representative blots. $\alpha$-tubulin was loading control. C. Densitometry analysis of PD-LI protein levels in the transplanted tumor cells in four groups. ${ }^{*} p<0.05$. Data represented mean \pm SD $(n=3)$.

\section{Discussion}

Radiotherapy is the primary treatment for lung cancer, and our results showed that radiotherapy can inhibit tumor growth and prolong the survival of tumor-bearing mice. We also demonstrated that compared with radiotherapy alone, radiotherapy combined with Fuzi remarkably inhibited tumor growth, increased tumor cell apoptosis and prolonged the survival of mice, indicating that Fuzi has a radiosensitizing effect.

To understand the mechanism underlying the radiosensitization effect of Fuzi, we first investigated the impact of Fuzi on immune function in mice after irradiation because accumulating data indicate that ionizing radiation influence antitumor immune response. IL-2 and IL-6 are known to be critical inducers of Th17 cell differentiation, which can enhance the body's immune response to prevent tumor growth [12]. We found that serum levels of IL-2, IL-5, IL-6, IL-12 in mice decreased significantly after radiation therapy. However, after additional treatment with Fuzi, serum levels of IL-2, IL-5, IL-6, IL-12 increased, which may promote the body's immune response and inhibit tumor growth. IL-10 and TGF- $\beta$ exert anti-inflammatory effects, promote Treg generation, and suppress the release of macrophage and monocyte produced inflammatory cytokines [13, 14]. These cytokines have been confirmed to promote invasion and metastasis of tumors in animal models. In present study, treatment with radiotherapy combined with Fuzi reduced the production of IL-10 and TGF- $\beta$ induced by radiation, which may explain how Fuzi regulates immune response to inhibit tumor growth and progression.

The physiological function of regulatory $\mathrm{T}$ (Treg) cells is the maintenance of tolerance to self-antigens and the suppression of autoimmune diseases, but Treg cells inhibit the development of efficient anticancer immune response [15, 16]. Tregs preferentially increase after radiation, and Tregs in a tumor context may assist in immune evasion during radiotherapy. Previous studies showed that IL-10 and TGF- $\beta$ induced mouse CD4 cells to become Tregs [17]. In this study, the proportion of Tregs increased after radiation but was decreased after radiotherapy combined with Fuzi. In addition, we observed coincidental changes in Treg proportions and TGF- $\beta$ and IL-10 levels. It is possible that Fuzi decreases the production of TGF- $\beta$ and IL-10 and thus reduces the production of Tregs.

Immune escape is the primary reason for the occurrence, development and recurrence of lung cancer, and PD-L1, a member of the co-stimulatory molecule B7 super family, is one of the important molecules involved in tumor immune escape [18]. In present study, radiotherapy promoted PD-L1 mRNA and protein expression in tumors, while Fuzi reduced local tumor PD-L1 expression. Previous studies showed that the interaction between PD-L1 and its receptor PD-1 on activated $\mathrm{T}$ cells impeded T-cell receptor signaling pathway that can lead to the differentiation into Foxp3 ${ }^{+}$Tregs, indicating that 
PD-L1 has a pivotal role in regulating Treg development [19-21]. In addition, studies have shown that PD-L1 expression by tumor cells may induce and maintain Tregs in tumor microenvironment, thereby augmenting the suppression of anti-tumor T-cell responses and allowing tumor progression [22]. However, the signaling pathways by which Fuzi acts on PD-L1 and modulates Tregs remain unclear and need further investigation.

In conclusion, we found that Fuzi combined with radiotherapy achieve significant anti-tumor effects, and Fuzi enhances immune response and weakens immune suppression and immune escape induced by radiotherapy. Fuzi is a promising radiosensitizer for adjunct therapy for lung cancer.

\section{Acknowledgments}

This work is supported by the Key Subject Construction Program of Jinshan District Health Administrative Authority (2012-24-733).

\section{Competing Interests}

The authors have declared that no competing interest exists.

\section{References}

1. Siegel RL, Miller KD, Jemal A. Cancer Statistics, 2017. CA Cancer J Clin. 2017; 67: 7-30.

2. Ferlay J, Soerjomataram I, Dikshit R, Eser S, et al. Cancer incidence and mortality worldwide: sources, methods and major patterns in GLOBOCAN 2012. Int J Cancer. 2015; 136: E359-86.

3. Pan $\mathrm{X}$, Wang $\mathrm{R}$, Bian $\mathrm{H}$, et al. Overexpression of Inhibitor of Growth 4 Enhances Radiosensitivity in Non-Small Cell Lung Cancer Cell Line SPC-A1. Technol Cancer Res Treat. 2016; Epub ahead of print.

4. Kreth G, Pazhanisamy SK, Hausmann M, et al. Cell type-specific quantitative predictions of radiation-induced chromosome aberrations: a computer model approach. Radiat Res. 2007; 167: 515-25.

5. Lee SJ, Jang BC, Lee SW, et al. Interferon regulatory factor-1 is prerequisite to the constitutive expression and IFN-gamma-induced upregulation of B7-H1 (CD274). FEBS Lett. 2006; 580: 755-62.

6. Kachikwu EL, Iwamoto KS, Liao YP, et al. Radiation enhances regulatory $\mathrm{T}$ cell representation. Int J Radiat Oncol Biol Phys. 2011; 81: 1128-35.

7. Zhou G, Tang L, Zhou $X$, et al. A review on phytochemistry and pharmacological activities of the processed lateral root of Aconitum carmichaelii Debeaux. J Ethnopharmacol. 2015; 160: 173-93.

8. Liou SS1, Liu IM, Lai MC, et al. Comparison of the antinociceptive action of crude Fuzei, the root of Aconitum, and its processed products. J Ethnopharmacol. 2005; 99: 379-83.

9. Nesterova IuV, Povet'eva TN, Aksinenko SG et al. Evaluation of anti-inflammatory activity of extracts from Siberian plants. Vestn Ross Akad Med Nauk. 2009; 11: 30-4.

10. Zhuang P, Huang $\mathrm{Y}, \mathrm{Lu} \mathrm{Z}$, et al. cAMP-PKA-CaMKII signaling pathway is involved in aggravated cardiotoxicity during Fuzi and Beimu Combination Treatment of Experimental Pulmonary Hypertension. Sci Rep 2016; 6: 34903.

11. $\mathrm{Li} \mathrm{H}$, Sun $\mathrm{M}, \mathrm{Xu} \mathrm{J}$, et al. Immunological response in $\mathrm{H} 22$ transplanted mice undergoing Aconitum coreanum polysaccharide treatment. Int $\mathrm{J}$ Biol Macromol. 2013; 55: 295-300.

12. Gao T, Bi H, Ma S, et al. The antitumor and immunostimulating activities of water soluble polysaccharides from Radix Aconiti, Radix A,coniti Lateralis and Radix Aconiti Kusnezoffii. Nat Prod Commun. 2010; 5: 447-55.

13. Ye ZJ, Zhou Q, Gu YY, et al. Generation and differentiation of IL-17-producing CD4+ T cells in malignant pleural effusion. J Immunol. 2010; 185: 6348-54.

14. Nova-Lamperti E, Fanelli G, Becker PD, et al. IL-10-produced by human transitional B-cells down-regulates CD86 expression on B-cells leading to inhibition of CD4+T-cell responses. Sci Rep 2016; 6: 20044.

15. Carter NA, Vasconcellos R, Rosser EC, et al. Mice lacking endogenous IL-10-producing regulatory $B$ cells develop exacerbated disease and present with an increased frequency of Th1/Th17 but a decrease in regulatory T cells. J Immunol. 2011; 186: 5569-79.
16. Cvetanovich GL, Hafler DA. Human regulatory $\mathrm{T}$ cells in autoimmune diseases. Curr Opin Immunol. 2010; 22: 753-60.

17. Wang HY, Wang RF. Regulatory T cells and cancer. Curr Opin Immunol. 2007; 19: $217-23$.

18. Nishikawa H, Sakaguchi S. Regulatory $\mathrm{T}$ cells in tumor immunity. Int J Cancer. 2010; 127: 759-67.

19. Chen ZM, O'Shaughnessy MJ, Gramaglia I, et al. IL-10 and TGF-beta induce alloreactive CD4+CD25- $\mathrm{T}$ cells to acquire regulatory cell function. Blood. 2003; 101: 5076-83.

20. de Roock S, Hoeks SB, Meurs L, et al. Critical role for programmed death 1 signaling and protein kinase $\mathrm{B}$ in augmented regulatory T-cell induction in cord blood. J Allergy Clin Immunol. 2011; 128: 1369-71.

21. Amarnath S, Mangus CW, Wang JC, et al. The PDL1-PD1 axis converts human TH1 cells into regulatory $T$ cells. Sci Transl Med. 2011; 3: 111ra120. Francisco LM, Salinas VH, Brown KE, et al. PD-L1 regulates the development, maintenance, and function of induced regulatory T cells. J Exp Med. 2009; 206: 3015-29.

22. Francisco LM, Sage PT, Sharpe AH. The PD-1 pathway in tolerance and autoimmunity. Immunol Rev. 2010; 236: 219-42. 